\title{
Shall We Still Consider HRTEM Image Simulation to Interpret Micrographs Obtained Using Cs (and Cc) Corrected Electron Microscopes?
}

\author{
Pierre Stadelmann
}

Ecole Polytechnique Fédérale de Lausanne, Centre Interdisciplinaire de Microscopie Electronique, CH-1015 Lausanne, Switzerland.

Image simulation has been widely used to interpret HRTEM micrographs over the last decades [1, 2, 3, $4,5]$. It allows a fine understanding of most the unexpected features of the experimental micrographs. It has been shown that excellent contrast matching is also obtained when the Modulation Transfer Function (MTF) of the image acquisition system is taken into account, potentially resolving the so-called Stobbs factor dilemma [6]. Under standard experimental conditions, i.e. moderate specimen thickness, the introduction of inelastically scattered electron does, usually, not change nor improve the quality of the comparison. With the development of Cs (and Cc) corrected electron microscopes one can hope to get rid of the often tedious process of image simulation. As a matter of fact, image simulation takes into account several processes, the electron-matter interaction, the transfer of information by the microscope and, finally, its detection. The last 2 , in most cases, are very well controlled and characterized. What remains difficult to master (and in principle unknown) are the specimen crystallography, thickness and orientation, all factors having an uppermost importance for a correct description of its (dynamical) interaction with the electrons. It is shown here that image simulation is still a valuable tool even when the experimental micrographs are acquired using $\mathrm{Cs}$ (and $\mathrm{Cc}$ ) corrected electron microscopes. When the crystal structure is known, image simulation has, furthermore, the capability to help figure out the best experimental conditions and to validate experimental protocols. With modern personal computers and programming languages performing HRTEM image simulation is a matter of a few minutes.

In order to demonstrate the potential of image simulation a simple hexagonal structure has been selected, $\mathrm{CdCu}_{2}$, shown in Figure 1 a) (3 cells are drawn to enhance its $\mathrm{P} 63 / \mathrm{mmc}$ hexagonal structure). Imaged in the [001] direction the $\mathrm{Cd}$ and $\mathrm{Cu}$ atomic columns are well separated, the $\mathrm{Cd}$ columns made of $2 \mathrm{Cd}$ atoms while the $\mathrm{Cu}$ columns contain either 1 or $3 \mathrm{Cu}$ atoms. The model projection is shown in Figure 1 b). The projected potential, calculated using atom form factors tabulated by Weickenmeier and Kohl [7], is displayed in Figure $1 \mathrm{c}$ ). The main question in almost all cases is: what are the proper experimental conditions that will make the image directly interpretable and allow clear distinction of the various atomic columns, i.e., what are the proper settings for accelerating voltage and the optimum specimen thickness for HRTEM images [8]. It is straightforward to show that dynamical scattering cannot be neglected and constitutes the major difficulty when trying to interpret experimental micrographs. After only a $5 \mathrm{~nm}$ thick $\mathrm{CdCu}_{2}$ crystal, Figure $2 \mathrm{~b}$ ), the distribution of intensity of the kinematical SAED pattern is already lost. As a result the $\mathrm{Cd}(2)$ as well as $\mathrm{Cu}(3)$ atomic columns disappear already for a $\sim 10$ $\mathrm{nm}$ thick crystal (under best imaging conditions). This phenomenon is, for $\mathrm{CdCu}_{2}$, aggravated at low accelerating voltage. Consideration of the effect of being slightly off-axis and a comparison with simulated HAADF images will be presented as well. 

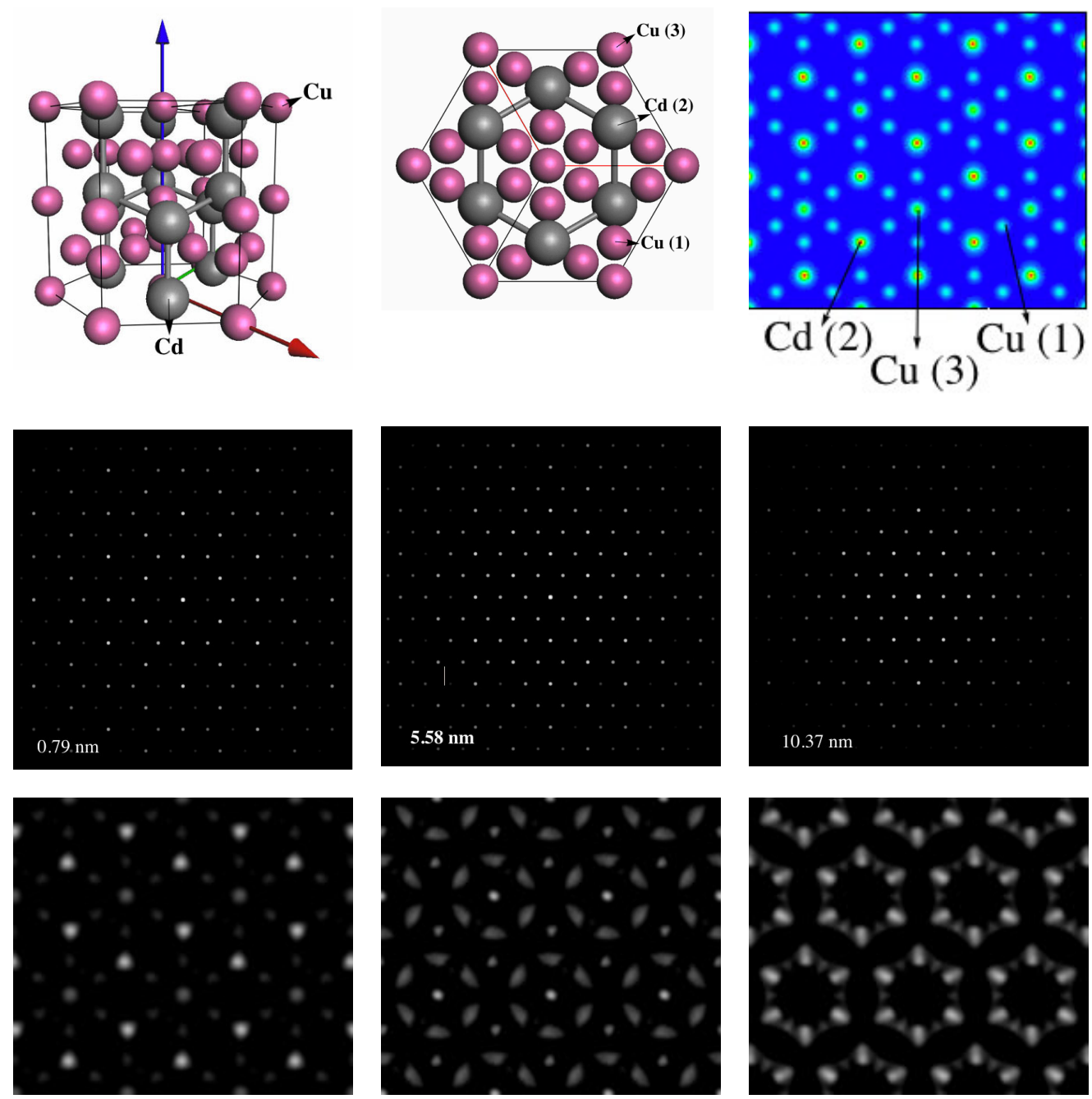

\section{References:}

[1] J. M. Cowley and A.F. Moodie, Acta Cryst. 10 (1957), 609.

[2] M. O'Keefe and J.V. Sanders, Acta Cryst. A31 (1975), 307.

[3] K. Ishizuka and N. Uyeda, Acta Cryst. A33 (1977), 740.

[4] W. M. J. Coene and D. van Dyck, Ultramicroscopy 15 (1984), 41.

[5] E.J. Kirkland in "Advanced Computing in Electron Microscopy” (Plenum Press, USA), 1998.

[6] A. Thust, 14th European Microscopy Congress proceedings (2008), 163.

[7] A. Weickenmeier and H. Kohl, Acta Cryst. A47 (1991), 590.

[8] C. Kübel and A. Thust, "True Image”, IFAM-Frauenhofer Institute (2005), 1.

Figure 1. $\mathrm{CdCu}_{2}$ a) model structure, b) $\mathrm{CdCu}_{2}$ [001] projection, c) projected potential. $\mathrm{Cd}$ or $\mathrm{Cu}$ atomic columns are marked as $\mathrm{Cd}(2), \mathrm{Cu}(1)$ and $\mathrm{Cu}(3)$ where the number of atoms in the atomic column is put in parentheses.

Figure 2. $\mathrm{CdCu}_{2}$ [001] simulated SAED diffraction patterns at $300 \mathrm{kV}, 249$ reflections, a) $0.79 \mathrm{~nm}$, b) $5.58 \mathrm{~nm}, \mathrm{c}) 10.37 \mathrm{~nm}$ thick.

Figure 3. $\mathrm{CdCu}_{2}$ [001] simulated HRTEM images at $300 \mathrm{kV}, 249$ reflections, a) $0.79 \mathrm{~nm}$, b) $5.58 \mathrm{~nm}$, c) $10.37 \mathrm{~nm}$ thick. 